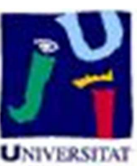

Título artículo / Títol article: Predictive Power Control for PV Plants With Energy Storage

Autores / Autors

E. Pérez, H. Beltrán, N. Aparicio,

P. Rodríguez

Revista:

Sustainable Energy, IEEE Transactions on

Versión / Versió:

Versió post-print

Cita bibliográfica / Cita

bibliogràfica (ISO 690):

PEREZ, Emilio, et al. Predictive power

control for PV plants with energy

storage. Sustainable Energy, IEEE

Transactions on, 2013, vol. 4, no 2, p. 482-

490.

url Repositori UJI:

http://hdl.handle.net/10234/89269 


\title{
Predictive Power Control for PV plants with Energy Storage
}

\author{
Emilio Pérez, Hector Beltran, Member, IEEE Néstor Aparicio, Member, IEEE \\ and Pedro Rodriguez, Senior Member, IEEE
}

SPECIAL ISSUE ON “APPLICATIONS OF SOLAR ENERGY TO POWER SYSTEMS”

\begin{abstract}
This work presents a model predictive control (MPC) approach to manage in real-time the energy generated by a grid-tied photovoltaic (PV) power plant with energy storage (ES), optimizing its economic revenue. This MPC approach stands out because, when a long enough prediction horizon is used, the saturation of the ES system (ESS) can be advanced by means of a prediction model of the PV panels production. Therefore, the PV+ES power plant can modify its production so as to manage the power deviations with regard to that committed in the daily and intraday electricity markets, with the objective of reducing economic penalties. The initial power commitment is supposed in this work to be given by a higher level energy management operator. By a proper definition of its objective function, the predictive control allows to economically optimize the $\mathrm{PV}+\mathrm{ES}$ power plant performance. This control strategy is tested in simulations with actual data measured for different days with varying meteorological conditions. Results provide a good reference on the economic benefits which can be obtained thanks to the MPC introduction.
\end{abstract}

Index Terms-Energy storage, photovoltaic systems, predictive control.

\section{INTRODUCTION}

G LOBAL warming and climate change are nowadays understood to be a serious problem for the planet which have much to do with electric power generation [1]. Thus, environmental together with geopolitical concerns are among the main reasons for the huge increase experienced worldwide in the use of renewable energy sources (RES) during the last decade [2], [3], with wind and solar power standing out in particular.

Solar photovoltaic (PV) power is one of the fastest-growing technologies in the RES domain. This is partially due to the support policies received in many countries in the form of premiums and feed-in tariffs. This trend will pose in brief a problem for the balance and stability of the power system in some countries [4] as a consequence of the variability of the solar irradiation [5], [6]. This intermittent character is therefore a clear drawback for this technology which, although being statistically predicted in an averaged mode, is stochastic in the short-term. Different solutions are already being implemented to mitigate this intermittency. These consider the geographical

This work was supported in part by the Universitat Jaume I under Grant P1.1A2008-11.

E. Pérez, H. Beltran and N. Aparicio are with the Area of Electrical Engineering, Universitat Jaume I, 12071 Castelló de la Plana, Spain (e-mail: pereze@uji.es).

P. Rodriguez is with the Electrical Engineering Department, Universitat Politècnica de Catalunya, 08222, Terrassa, Spain (e-mail: prodriguez@ee.upc.edu). dispersion of PV systems across regions to reduce clouds' effect [7], or the integration of energy storage (ES) systems with RES [8]-[12]. The latter empowers RES with extended capabilities such as production shifting [13], peak shaving or the possibility to provide ancillary services [14]. There are nowadays several ES technologies available in the market [14], [15]. All of them still expensive for PV applications though. Therefore, an accurate ES sizing calculation and an optimized control strategy for the PV+ES power plant turn to be key issues for the future economic viability of these hybrid plants. Thus, different proposals have been recently published to drive PV power plants with ES [9], [16]-[20]. However, most of them are focused on isolated grids and microgrids or rely on basic control approaches which require of a large ES system (ESS).

This paper presents an advanced control approach designed to manage in real-time the power production of a grid-tied $\mathrm{PV}+\mathrm{ES}$ power plant with a reduced ESS capacity which participates in the electricity market. The main contribution of this control proposal lies on the fact that it can anticipate future saturations of the ESS (likely to occur with reduced rating) and, therefore, modify the response of the hybrid power plant so as to minimize the economic penalties due to the power production deviations referred to the power committed in the electricity pool. This control approach is tested through simulation with actual data for various days with varying meteorological conditions and predictions with different degrees of accuracy.

The paper starts by describing the problem of integrating PV plants into the electricity market and with a proposal to solve it taking advantage of an advanced control methodology. Section III overviews some of the most important electricity markets configurations worldwide. After that, Section IV presents the control methodology with its mathematical formulation. Section V is devoted to the discussion of the results obtained when this control approach is applied. The differences in the power plant performances and the economic improvement achieved are analyzed. Finally, some conclusion remarks are discussed in Section VI.

\section{PROBLEM DESCRIPTION}

As just introduced, the work presented here focuses on defining a control strategy to manage the generation of a gridtied PV power plant which takes part in the electricity market. Therefore, the plant must regularly commit a constant-byhours power production with some hours before the real-time delivery instant. 
Given the non-controllable and stochastic nature of the solar resource, and hence that of its corresponding PV power production, a natural solution is the use of an ESS which allows accumulating the surplus energy in those periods in which solar production is higher than the plant power commitment and delivering it back in the opposite situation.

Although most of the work in the existing literature regarding the operation of $\mathrm{PV}+\mathrm{ES}$ systems focuses on isolated networks, several approaches [9], [12], [16], [18] are already devoted to grid-connected systems. Each of those proposals pursues different control objectives (mainly peakshaving). To the authors' knowledge, the field of grid-tied market participant $\mathrm{PV}+\mathrm{ES}$ power plants is still emerging. However, some contributions have been made for combined wind-storage systems in the electricity market which could be extended to other non-programmable RES such as PV. In [21], the authors use an optimization scheme similar to the one proposed here, although solved by dynamic programming. In [22], an iterative methodology, based on technical or marketbased criteria, is proposed. Although related to our proposal to some extent, those works still have a crucial difference: they focus mainly on the day-ahead scheduling stage, and not on the real-time operation. For this stage, all those works share a common strategy, i.e. they define a power reference to the plant, $P_{r e f}$, and compute the power exchange with the ESS, $P_{E S}$, according to the following equation:

$$
P_{E S}(t)=P_{r e f}(t)-P_{P V}(t)
$$

where $P_{P V}$ is the actual PV panels production. If the computed $P_{E S}(t)$ exceeds the admissible power or if it produces the ESS saturation, it is reduced to an adequate value.

The described strategy is valid for the control objectives defined on those works, but for the problem considered in this paper, it brings some difficulties. This is so because the power plants attending the electricity market must provide a power commitment in advance, when the actual PV production during the power plant operation is still not known with precision. Thus, it can only be approximated with a certain degree of accuracy. In this context, when the delivery time arrives, it might happen that, even with the presence of the ESS, the committed power cannot be achieved because of the ESS saturation. This fact forces the PV+ES plant to feed directly to the grid the power generated in the PV panels, incurring an economic penalty. Therefore, with the basic strategy described in (1), this event occurs at a non-controlled moment, which can be very detrimental from the economic point of view (because of the energy cost during that period of time).

In this paper, a different strategy based on the Model Predictive Control (MPC) philosophy is proposed to control these systems. This proposal substitutes the basic approach in (1) and computes $P_{E S}(t)$ not only considering the $P_{\text {ref }}(t)$ and $P_{P V}(t)$ at that given moment, but also taking into account what are the future references, $P_{\text {ref }}(t+k)$, the predicted PV power production, $\hat{P}_{P V}(t+k)$, and the future energy prices in the electricity market, Fig. 1. As this MPC-based strategy is performed in real-time, it can use much more accurate shortterm PV power predictions. This characteristic enables the control system to detect ESS saturation in advance and modify

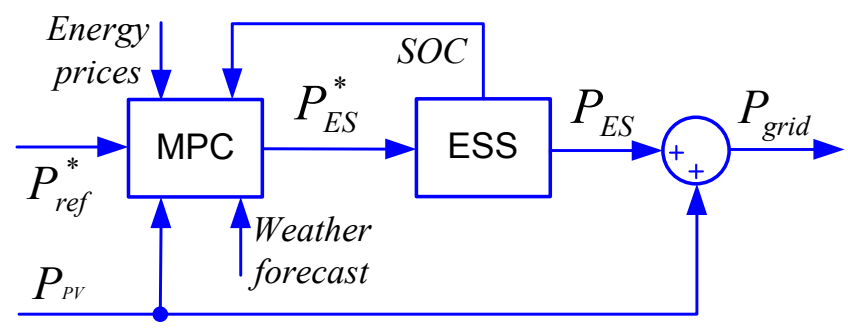

Fig. 1. Model Predictive Control scheme.

the operation of the power plant so as to optimally reduce economic penalties.

The application of MPC has already been treated in the context of RES. In [23] and [24], authors proposed an electric energy system integrating various RES (combining PV and wind installations) and used the MPC to optimize an economic objective function with the possibility to include also environmental costs. Those proposals differ substantially from the one presented here, since the goal in those cases is to use the RES to respond quickly to sudden increases in the power system demand, avoiding the use of high-price coal plants. This implies that the energy from the RES is not fully profited, contrary to the goal of the present work. Furthermore, there is no ES introduction and the PV power prediction is done in a period too short to be used with market participation goals.

A more similar approach to the one proposed in this work is found in [25], where the authors propose an analogous formulation applied to a wind farm with ES. However, the control target in that work is not to enable the system to participate in the electricity market, but to smooth the wind production. Furthermore, the stochastic nature of wind power production only allows forecasting a few steps ahead, which is not compatible with market participation. In this sense, the different electricity market designs highly influence the problem presented here. That is the reason for the market analysis introduced in the following.

\section{MARKET PARTICIPATION}

In order to properly test the PV+ES operation improvement achieved with this new control approach, it is important to know the electricity market structure and its scheduling configuration. These mainly depend on the different countries and their corresponding regulations. In this sense, countries with restructured electricity industries usually have forward markets where, depending on the market design, electricity is traded either centralized on a power exchange or bilaterally directly. Electricity is traded in intervals (hereinafter referred to as settlement periods) that may be one hour long or less. Market participants can modify their bids and offers for any settlement period before the gate closure. After that point it is no longer possible to change the energy committed for certain coming settlement periods. Each settlement period is settled independently of the periods around it. Some regions run continuous markets with gate closures some minutes before actual delivery (5 min in Australia, $60 \mathrm{~min}$ in the United Kingdom and in some U.S. markets). On the contrary, other regions have daily markets (especially suited for the unit 
commitment of thermal generators) that close in the day-ahead of the energy delivery.

During real-time, i.e. after gate closure, generators production may differ from the one committed in the market and also demand may change. Thus, system operators must continuously balance the sum of all individual imbalances (system net imbalance) through balancing mechanisms. The cost of the regulating energy required by the balancing mechanisms is usually assigned to those market participants who are responsible for the net imbalance. This mechanism mainly affects intermittent energy sources due to their inherent unpredictability.

Therefore, market design has a clear influence in the imbalance settlement of intermittent energy sources such as PV. In this way, this will vary depending on the possibilities to reschedule production with updated forecasts, the price system or whether RES are fully charged for their imbalances.

Among the different market configurations, the Spanish market has been selected for the simulations performed in this work. It has some particular characteristics such as a special intraday market (which permits to reschedule with updated production forecasts in the next hours) that instead of being continuous is divided into six sessions. On each of these sessions, the power exchange of the daily market is repeated for the settlement periods still to come in the delivery day. In addition, the Spanish market has a two-price system for the imbalances settlement. The imbalance price is the market price for producers that do not contribute to the system net imbalance and a penalty for those that do contribute. Moreover, since 2007, all generators (including RES) are fully charged even if they do not participate in the electricity market (i.e. receive feed-in tariffs).

\section{Model Predictive Control applied to PV PLANTS WITH ENERGY STORAGE SYSTEM}

\section{A. Model Predictive Control}

Model Predictive Control (MPC) is a controller design technique based on the following strategy [26]:

1) The future outputs for a given horizon $N$, called the prediction horizon, are predicted at each instant $t$ using the process model. These predicted outputs $y(t+k \mid t)$ depend on the known values of past inputs and outputs and on the future control signals $u(t+k \mid t)$.

2) These control signals are calculated by optimizing a determined criterion dependent on the predicted future trajectory and control signals.

3) Although a complete sequence of $N$ future control signals is computed, only $u(t \mid t)$ is effectively sent to the process, because at the next sampling instant a new output $y(t+1)$ is known. This is known as receding horizon.

MPC presents a series of advantages over other control methods [26], [27]: multivariable cases can be systematically designed by assigning a different relative importance to each input and output, allowing an intuitive tuning of the controller; constraints can automatically be taken into account and, when future references or disturbances are known, this information can be used by the controller, allowing a performance improvement.

On the contrary, the main drawbacks of MPC are the need for an accurate prediction model of the controlled outputs and the computational effort required to solve a constrained optimization problem, which can be too consuming for fast process applications.

\section{B. Problem formulation}

The problem described in Section II can be approached by means of an MPC strategy. The main idea is to maximize a given economic objective function, $J_{N}$, defined within a future horizon $N$, while the problem constraints are satisfied.

Unlike for the case of the basic subtraction strategy (1), if predictive control is applied to the system, the prediction of the future solar production is updated in real-time. Therefore, limitations to track future power commitments can be detected in advance, making it possible to shift the power reference tracking failure to other moments when economic penalties are lower.

Similarly, if the solar production is higher than expected and this fact is detected in advance by the MPC system, the energy exceeding the plant production commitment can be fed to the grid during those periods when the energy market price is higher.

In accordance with the previous considerations and the MPC formulation, the objective function to be maximized can be written as:

$$
\begin{aligned}
J_{N}= & \sum_{k=0}^{N} \lambda(k) c(t+k) T\left(P_{\text {grid }}(t+k)-P_{\text {ref }}(t+k)\right) \\
& +c_{s o c}(t+N+1) E_{E S}(t+N+1)
\end{aligned}
$$

where:

- $P_{r e f}(t+k)$ is the future constant-by-hours power production committed by the PV plant.

- $P_{\text {grid }}(t+k)$ is the power fed to the grid at instant $(t+k)$, that is $P_{\text {grid }}(t+k)=P_{P V}(t+k)+P_{E S}(t+k)$, being $P_{P V}(t+k)$ the panel production and $P_{E S}(t+k)$ the power exchanged with the ESS.

- $E_{E S}(t+N+1)$ is the energy stored in the ESS at instant $(t+N+1)$.

- $c(t+k)$ is the imbalance cost at instant $(t+k)$.

- $c_{\text {soc }}(t+N+1)$ is the value of the energy stored in the ESS at instant $(t+N+1)$.

- $\lambda(k)$ is a weighting sequence.

- $T$ is the sampling period of the MPC.

Note that the energy stored in the ESS at any future instant $E_{E S}(t+k)$ can be calculated as:

$$
E_{E S}(t+k)=E_{E S}(t+k-1)-T P_{E S}(t+k-1)
$$

The objective function (2) consists of two terms: a weighted summation of the cost of the future power deviations referred to the plant commitments in the prediction horizon and a term which values the energy stored in the ESS after that horizon.

The first term aims to value the economic revenues obtained from the power fed to the grid in the prediction horizon. As 
described in Section III, in the Spanish electricity market the system operator settles imbalances by means of a two-price system. Therefore, the imbalance cost at any given instant must adopt different values depending on the imbalance sign:

$$
c(t+k)= \begin{cases}c_{p}(t+k) & \text { if } P_{\text {grid }}(t+k) \geq P_{\text {ref }}(t+k) \\ c_{n}(t+k) & \text { if } P_{\text {grid }}(t+k)<P_{\text {ref }}(t+k)\end{cases}
$$

where $c_{p}$ and $c_{n}$ are the constant-by-hours positive and negative imbalance costs, respectively.

The different summands in this term are adjusted by means of a weighting sequence defined as $\lambda(k)=\alpha^{-k}$, with $\alpha$ taking a value between 0 and 1 . The introduction of such a sequence is useful because otherwise the optimization could present multiple solutions with the same maximum, due to the constant-by-hours nature of the costs. The proposed weighting sequence, which increases with $k$, gives more relevance to the economic benefits obtained in instants of time further from the current moment than to those obtained in closer instants. The reason for this is that, as will be discussed in Section IV-D, the prediction of the solar production is more accurate as time goes by, and therefore, it is more convenient to save up energy for future moments which keep the same imbalance costs.

Regarding the second term of the objective function (2), it is introduced in the optimization in order to take into account that the remaining energy in the ESS after the control horizon still presents a value. Otherwise, the optimization would always tend to empty the ESS, which obviously is not always the optimal strategy. However, it is not straightforward to determine this energy value, $c_{s o c}(t+N+1)$, as there are many different prices depending on the future instant of time in which this amount of energy would be sold. Furthermore, it is also difficult to decide whether this energy should be valued at a positive or negative imbalance price, as future imbalance signs are unknown a priori. If positive imbalance prices are taken, the optimization will generally tend to discharge the ESS. Conversely, if negative imbalance prices are chosen, it will be more conservative. Note that with the latter, the optimization might even decide to produce a negative imbalance during the optimization horizon in order to avoid a potentially more expensive imbalance after it. As this is not a desirable behavior, the energy stored is valued at a positive imbalance price, i.e. $c_{s o c}(t+N+1)=c_{p}(t+N+1)$.

Apart from the objective function, it is also necessary to define some constraints on the power to be fed to the grid in future instants:

- For an efficient performance of the ESS, its state of charge (SOC) must be kept between a minimum and a maximum level: $E_{E S, \text { min }}$ and $E_{E S, \text { max }}$.

- The power exchanged by the system with the ESS (in both possible directions) is limited by the converter's rated power capacity, $P_{E S, \max }=-P_{E S, \min }$.

The proposed predictive controller can be obtained by solving the optimization problem (5), which includes the previous constraints to be satisfied in all the future instants, together with a receding horizon strategy (applying only the first power exchange with the ESS, $P_{E S}(t)$, and solving a new optimization problem every time new measured data are received).

$$
\begin{aligned}
& J_{N}^{\text {opt }}=\max J_{N} \\
& \text { subject for } k=0 \ldots N \text { to: } \\
& P_{\text {grid }}(t+k)=\hat{P}_{P V}(t+k)+P_{E S}(t+k) \\
& E_{E S}(t+k+1)=E_{E S}(t+k)-T P_{E S}(t+k) \\
& P_{E S, \text { min }} \leq P_{E S}(t+k) \leq P_{E S, \max } \\
& E_{E S, \text { min }} \leq E_{E S}(t+k) \leq E_{E S, \text { max }}
\end{aligned}
$$

Note that all variables included in the constraints of the system and in the objective function are known in advance, except for the future solar production, $P_{P V}(t+k)$, and the future imbalance and energy costs, $c_{p}, c_{n}$ and $c_{\text {soc }}$. Therefore, models to predict each of them are needed. Discussion on these models will be performed in following subsections.

\section{Optimization problem}

The application of the proposed MPC involves solving, at every sampling instant, the constrained optimization problem (5) in order to compute the power to be exchanged with the ESS. This is the most demanding operation in terms of computational cost for any predictive controller and the one that can prevent the use of this technique for applications with low sampling periods.

This computational cost mainly depends on the size and kind of problem, determined by the objective function and the constraints. In this case, the size of the problem under consideration is determined by the control horizon, $N$. On the other hand, the system constraints are linear, being the simplest kind of constraints, but the objective function is neither linear nor quadratic, making the problem difficult to solve in its straightforward form. This is due to the fact that the imbalance cost (4) is a piecewise function, which depends on the sign of the power deviation.

One possibility to solve this problem is to formulate it as a hybrid system, introducing a binary variable to cope with the piecewise objective function. This kind of problems has been an active research topic in the field of predictive control in the recent years [28]. However, for a general case, they require to solve a mixed integer linear program (MILP) which would be computationally prohibitive for the size of the proposed problem.

Therefore, an alternative approach, based on transforming the objective function into a linear one, is proposed. The main idea is to substitute the variable $P_{\text {grid }}(t+k)$ by two different ones, $P_{p}(t+k)$ and $P_{n}(t+k)$, which replace $P_{\text {grid }}$ for the cases when this is higher or lower than $P_{r e f}(t+k)$, respectively:

$$
P_{\text {grid }}= \begin{cases}P_{p} & \text { if } P_{\text {grid }} \geq P_{\text {ref }} \\ P_{n} & \text { if } P_{\text {grid }}<P_{\text {ref }}\end{cases}
$$

In order to avoid this piecewise representation, the same equation can be written as a linear expression with the 
appropriate constraints:

$$
\begin{aligned}
& P_{\text {grid }}(t+k)=P_{p}(t+k)+P_{n}(t+k)-P_{\text {ref }}(t+k) \\
& P_{p}(t+k) \geq P_{\text {ref }}(t+k) \\
& P_{n}(t+k) \leq P_{r e f}(t+k) \\
& \left(P_{p}(t+k)-P_{\text {ref }}(t+k)\right) \cdot\left(P_{n}(t+k)-P_{r e f}(t+k)\right)=0
\end{aligned}
$$

where (10) is introduced to force that either $P_{p}(t+k)=$ $P_{\text {ref }}(t+k)$ or $P_{n}(t+k)=P_{\text {ref }}(t+k)$ and, therefore, from (7) $P_{\text {grid }}(t+k)=P_{n}(t+k)$ or $P_{\text {grid }}(t+k)=P_{p}(t+k)$, respectively. This transformation allows to rewrite the objective function as a linear one:

$$
\begin{aligned}
J_{N}^{\prime}= & \sum_{k=0}^{N} \alpha^{-k} T\left[c_{p}(t+k)\left(P_{p}(t+k)-P_{r e f}(t+k)\right)\right. \\
& \left.+c_{n}(t+k)\left(P_{n}(t+k)-P_{r e f}(t+k)\right)\right] \\
& +c_{s o c}(t+N+1) E_{E S}(t+N+1)
\end{aligned}
$$

With this new formulation, the optimization problem (5) can be rewritten as:

$$
J_{N}^{o p t}=\max J_{N}=\max J_{N}^{\prime}
$$

subject for $k=0 \ldots N$ to:

$$
\begin{aligned}
& E_{E S}(t+k+1)=E_{E S}(t+k)-T P_{E S}(t+k) \\
& P_{E S, \text { min }} \leq P_{E S}(t+k) \leq P_{E S, \max } \\
& E_{E S, \text { min }} \leq E_{E S}(t+k) \leq E_{E S, \text { max }} \\
& P_{p}(t+k)+P_{n}(t+k)-P_{r e f}(t+k) \\
& \quad=\hat{P}_{P V}(t+k)+P_{E S}(t+k) \\
& P_{p}(t+k) \geq P_{r e f}(t+k) \\
& P_{n}(t+k) \leq P_{r e f}(t+k) \\
& \left(P_{p}(t+k)-P_{r e f}(t+k)\right) \cdot\left(P_{n}(t+k)-P_{r e f}(t+k)\right)=0
\end{aligned}
$$

At this point, the original problem with a non-linear objective function and linear constraints has been transformed into an equivalent problem with a linear objective function, several linear constraints and a single non-linear constraint, (10). This new problem may not be simpler than the original one in a general case. However, it is interesting to consider now the particular problem under study. To do so, let us first calculate $E_{E S}(t+N+1)$ by recursive application of (3) and substitution of (13):

$$
\begin{aligned}
& E_{E S}(t+N+1)= E_{E S}(t)-T \sum_{k=0}^{N} P_{E S}(t+k) \\
&=E_{E S}(t)-T \sum_{k=0}^{N}\left(P_{p}(t+k)+P_{n}(t+k)\right. \\
&\left.-P_{r e f}(t+k)-\hat{P}_{P V}(t+k)\right)
\end{aligned}
$$

Substituting on (11) and grouping terms:

$$
\begin{aligned}
J_{N}^{\prime}= & \sum_{k=0}^{N} T\left[\left(\alpha^{-k} c_{p}(t+k)-c_{s o c}(t+N+1)\right) P_{p}(t+k)\right. \\
& \left.+\left(\alpha^{-k} c_{n}(t+k)-c_{s o c}(t+N+1)\right) P_{n}(t+k)\right]+C
\end{aligned}
$$

where $C$ contains all the terms in $J_{N}^{\prime}$ which do not depend on the decision variables, $P_{p}(t+k)$ and $P_{n}(t+k)$.

Now note that, $c_{n}(t+k)>c_{p}(t+k)$ at any moment because of the nature of the electricity markets, as it has been discussed in Section III. Indeed, $c_{n}(t+k)$ is the price at which a market participant has to buy energy to the system if it is not able to supply all the energy committed, and therefore it is paid at a price higher than the market one $\left(c_{m}(t+k)\right)$. Conversely, $c_{p}(t+k)$ is the price at which the extra energy exceeding the commitment has to be sold to the market, which is therefore lower than the market one. Consequently, for the same instant $(t+k)$ :

$$
c_{n}(t+k) \geq c_{m}(t+k) \geq c_{p}(t+k)
$$

From (14) and (15) it can be deduced that, whenever it is possible, it is more beneficial to increase $P_{n}(t+k)$ than $P_{p}(t+k)$. Now, observe that, apart from constraints (8), (9) and (10), both variables appear always as a summation. This means that, when regarding the charging and discharging of the ESS, it is the same to increase one or the other. Obviously, as $P_{n}$ produces a higher increase in the objective function, where possible, the optimizer will leave $P_{p}$ at its minimum, $P_{p}=P_{\text {ref }}$. On the other hand, if it is not possible to satisfy all the constraints, the optimizer will leave $P_{n}$ at its maximum, $P_{n}=P_{\text {ref }}$, and will start increasing $P_{p}$.

This means that, by the same nature of the problem, constraint (10) is implicitly satisfied when the rest of constraints are met, thus it is redundant. Therefore, it can be eliminated from the formulation of the problem. Note that this mathematical fact has a physical interpretation. If, for the same instant of time $(t+k)$, both $P_{p}(t+k)$ and $P_{n}(t+k)$ were different than $P_{\text {ref }}(t+k)$, there would be simultaneously a positive and a negative imbalance.

After dropping constraint (10) from (12), the optimization problem to be solved by the predictive controller can be finally defined as a linear program (LP), which is easily solvable with standard optimization tools.

\section{Solar production prediction}

In order to define a proper $\hat{P}_{P V}$ model which assures an optimal problem resolution, standard irradiation curves provided by official databases such as the PVGIS [29] are used. These are continuously adjusted by the actual PV production measured till the moment of calculation. Given that PVGIS data are statistically-averaged during the years and provide monthly-averaged irradiation profiles, these have to be adjusted every day to adapt them to the actual meteorological conditions and, thus, generate a realistic $\hat{P}_{P V}$ model for that day. Here is proposed an adjustment based on the real-time calculation of the PV energy produced by the panels throughout the day until a given instant of time, $\left(E_{P V}(t)\right)$ and on its comparison with the ideal energy $\left(E_{P V G I S}(t)\right.$, according to the PVGIS model for real sky conditions) which should be expected at any moment [30]. The quotient among these two energy values provides an instantaneous daily weatherdependent coefficient which varies throughout the daytime. This parameter has been called Cloudiness Coefficient $(C C)$ 


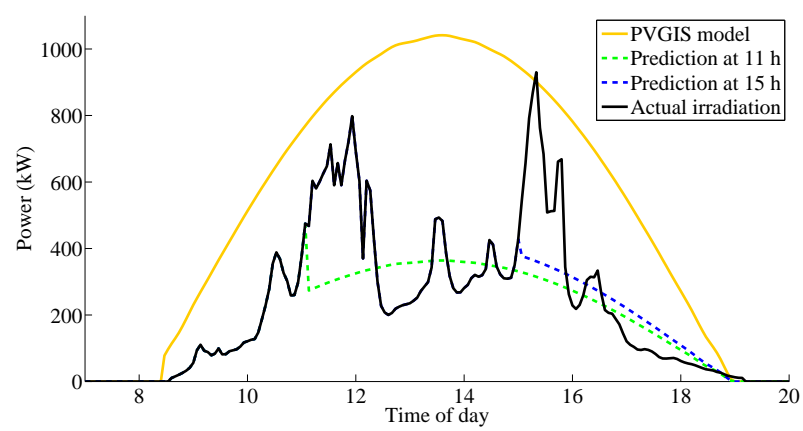

Fig. 2. Solar irradiation on the panels and predictions. Cloudy day.

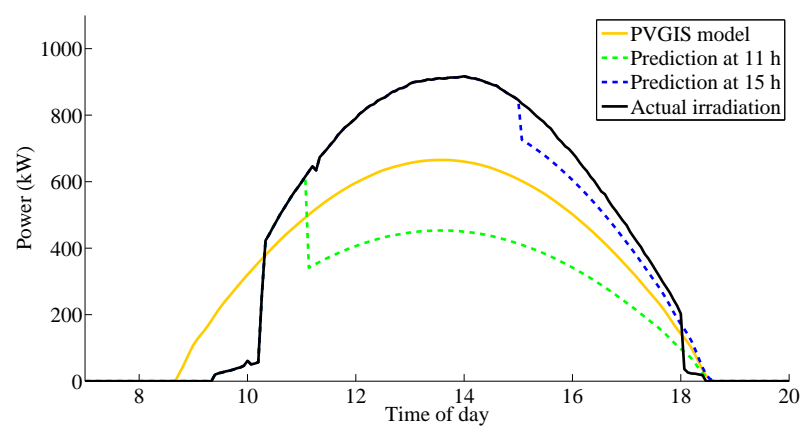

Fig. 3. Solar irradiation on the panels and predictions. Sunny day.

and is defined as:

$$
C C(t)=\frac{E_{P V}(t)}{E_{P V G I S}(t)}
$$

As the day goes on, $C C(t)$ gets more stable and closer to the value that it will take at the end of the day. Combining this fact with the well-known profile of the production according to PVGIS, which exactly defines $E_{P V G I S}(t)$, the prediction model is capable to progressively estimate $\hat{P}_{P V}(t+k)$ more accurately according to the following equation:

$$
\hat{P}_{P V}(t+k)=C C(t) \cdot P_{P V G I S}(t+k)
$$

Fig. 2 shows the proposed PV power prediction for a cloudy day at two different instants of time, $11 \mathrm{~h}$ and $15 \mathrm{~h}$. Both predictions given by the proposed model are quite accurate. On the other hand, Fig. 3 shows the PV power prediction at the same instants of time for a sunny day with some clouds during dawn. In this case, the prediction at $11 \mathrm{~h}$ is quite far under the actual PV production. However, the prediction at $15 \mathrm{~h}$, is much more accurate because as it is later in the daytime, $C C$ is more precise.

It is important to notice that, because of the nature of the problem and the proposed controller, it is not necessary to have a very accurate pointwise-in-time prediction of the PV production, but rather an estimation of the energy given by the PV panels in a period of time, i.e. the area under the power curves. Therefore, the proposed prediction model is useful.

Note also that more complex weather forecasting systems, such as satellite images [31] or Doppler radar systems [32], [33], could be incorporated to the proposed MPC philosophy, as long as they can provide a numerical PV production prediction at every sampling time. However, the analysis and

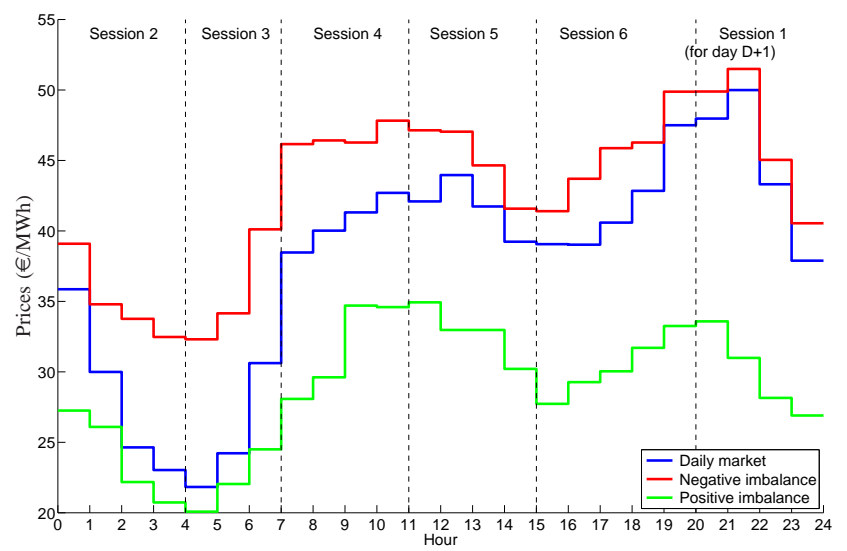

Fig. 4. Average energy prices in the Spanish market.

efficiency of the weather forecast techniques are beyond the scope of this paper.

\section{E. Imbalance settlement prices}

Finally, the last inputs necessary for the MPC implementation are the estimation of the imbalance settlement prices. Liberalized electricity markets have an effective public information policy for both market participants and the general public in order to ensure the transparency of the system operator's actions and, therefore, the electricity prices are public and easy to find out. That is the case of the Spanish market, explained in Section III, which uses a two-price system for imbalance settlements. The Spanish system operator provides relevant information about market results, including hourly prices of daily and intraday markets, and positive and negative imbalance settlements [34]. As starting estimation, imbalance costs (4) are considered for the design of the controller as the yearly-averaged price for each settlement period, shown in Fig. 4 for year 2010. However, market participants with improved information of electricity prices and data processing could employ different estimation models that could take into account particular circumstances such as seasonal trends, holidays, and other kind of singular events.

\section{RESUlts}

The proposed MPC strategy has been tested by simulation using actual irradiation data measured with a sampling period $T=4 \mathrm{~min}$ in a PV installation located in Seville (Spain). The nominal power of both the PV power plant and the ESS power converter is $500 \mathrm{~kW}$ and the considered ESS has an energy capacity of only $0.8 \mathrm{MWh}$, which is likely to saturate.

The MPC tuning parameters are given in Table I, and the $\mathrm{PV}$ production is predicted by $\hat{P}_{P V}$ as described in Section IV-D. Three case studies are proposed: one in which the PV power production is overestimated in the moment that power commitments are settled, other in which this production is underestimated, and a third one with an accurate estimation. In each of them, the MPC strategy is compared with the basic substraction strategy (1) in terms of obtained revenues. These 
TABLE I

MPC TUNING PARAMETERS

\begin{tabular}{|c|c|c|c|c|}
\hline $\boldsymbol{N}$ & $\boldsymbol{T}$ & $\boldsymbol{\lambda}(\boldsymbol{k})$ & $\boldsymbol{c} \boldsymbol{p}$ & $\boldsymbol{c}_{\boldsymbol{n}}$ \\
\hline 60 & $4 \mathrm{~min}$ & $0.999^{k}$ & as in Fig. 4 & as in Fig. 4 \\
\hline \hline $\mathbf{c}_{\mathbf{s o c}}(\mathbf{t})$ & $\mathbf{P}_{\mathbf{E S}, \min }$ & $\mathbf{P}_{\mathbf{E S}, \max }$ & $\mathbf{E}_{\mathbf{E S}, \min }$ & $\mathbf{E}_{\mathbf{E S}, \max }$ \\
\hline$c_{p}(t)$ & $-500 \mathrm{~kW}$ & $500 \mathrm{~kW}$ & 0 & $0.8 \mathrm{MWh}$ \\
\hline
\end{tabular}
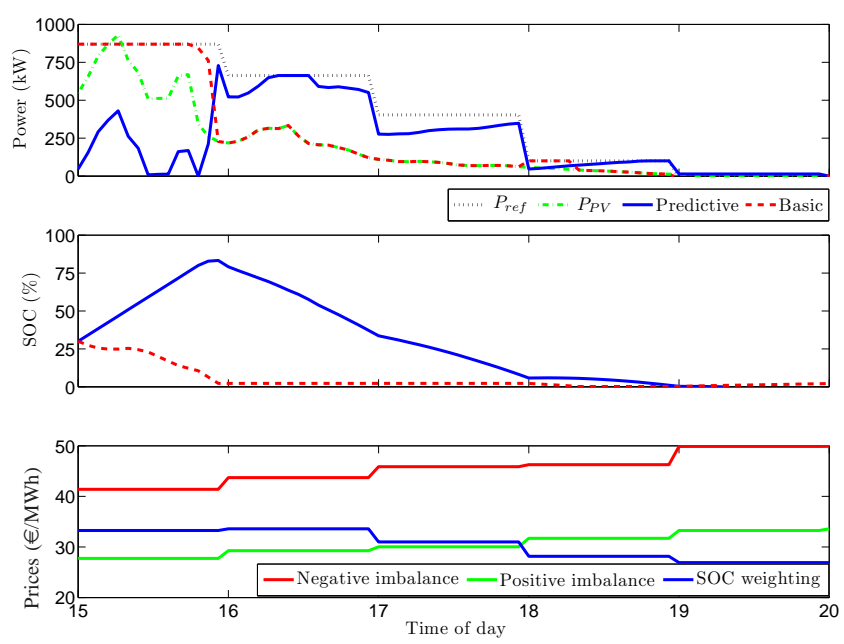

Fig. 5. Overestimation of PV production case study.

are calculated considering the imbalance costs (4) and the energy cost $c_{\text {soc }}(t)=c_{p}(t)$, as discussed previously.

$$
\begin{aligned}
R= & \sum_{t=0}^{t_{s i m}} T\left(c(t)\left(P_{\text {grid }}(t)-P_{r e f}(t)\right)\right) \\
& +T c_{s o c}\left(t_{\text {sim }}+1\right) E_{E S}\left(t_{s i m}+1\right)
\end{aligned}
$$

where $t_{\text {sim }}$ is the final simulation instant.

All simulations are implemented in MATLAB in an Intel Pentium D CPU 2.66GHz with $1 \mathrm{~GB}$ of RAM. The LPs are solved by the Double Description algorithm [35], by means of the MATLAB interface to the CDD software package [36] provided by the MPT Toolbox [37].

\section{A. Overestimation of $P V$ production}

As a first case study, the period of time corresponding to sixth session of the intraday market has been considered for the cloudy day which PV production is shown in Fig. 2. For that day, the energy management strategy implemented to define the power commitments of the PV+ES plant overestimates the $\mathrm{PV}$ production of the panels as indicated by the PVGIS model curve and, therefore, it generates a set of future references, $P_{r e f}(t+k)$, higher than those that the system is capable to produce. The ESS is initially at $30 \%$ SOC. Fig. 5 shows the obtained results.

From Fig. 5, the advantages of the MPC strategy become apparent. The basic strategy starts following exactly $P_{\text {ref }}$, adding to the insufficient PV production the power from the ESS, which discharges in less than one hour. On the other hand, the MPC predicts soon that the ESS will discharge if the committed $P_{r e f}$ are followed. Taking into account the price structure, the control system including MPC tends to keep
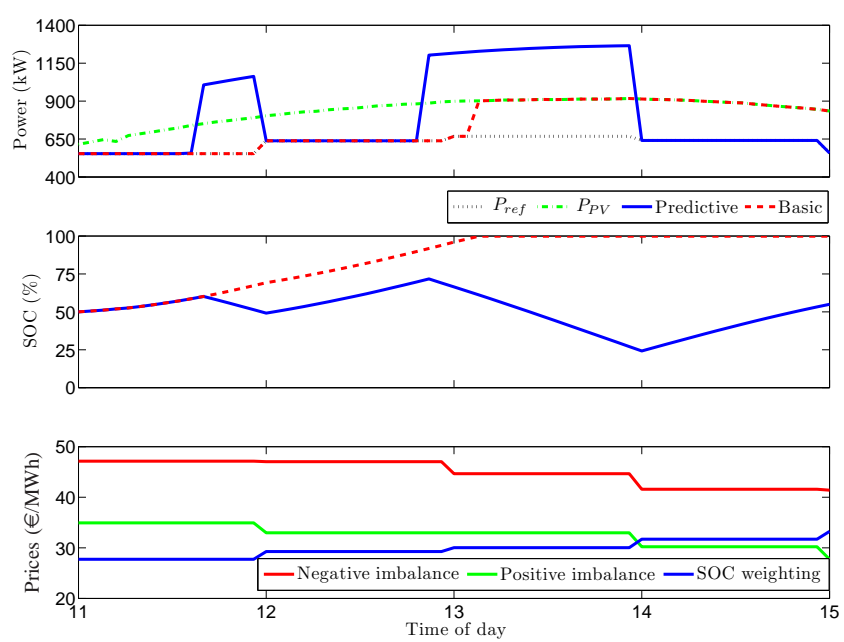

Fig. 6. Underestimation of PV production case study.

energy in the ESS in order to track the reference during the latest hours of the intraday market session, when the imbalance penalties are higher. That is, the MPC allows the system to reduce its power production during the first hours in order to charge the ESS. Moreover, it can be observed how the ESS finishes the session completely discharged (as in the basic strategy). Note that this is indeed the optimal behavior, as power commitments after $20 \mathrm{~h}$ were settled to 0 because of the PVGIS prediction, and therefore any surplus of energy would have to be sold at a (much lower) positive imbalance price.

If economic revenues are calculated following (16) for both strategies, MPC's is found to be $4.4 \%$ higher, because tracking failure is shifted to less expensive periods.

Simulation for the 5-hour period lasted a total time of $251.6 \mathrm{~s}$ in the aforementioned hardware, with an average and a maximum computation time for the optimizations of, respectively, $3.26 \mathrm{~s}$ and $3.45 \mathrm{~s}$. The MPC strategy with $N=60$ (and with higher horizons) is therefore fast enough to be implemented with the proposed sampling period of $4 \mathrm{~min}$.

\section{B. Underestimation of $P V$ production}

In the second case study, we analyze the opposite situation. That is, during the sunny day whose PV production is shown in Fig. 3, the energy management system underestimates the PV production as shows the PVGIS model curve. The working period corresponds in this case to the fifth session of the intraday market and the ESS is initially at $50 \%$ SOC. The performance of the basic and the MPC strategies are compared in Fig. 6.

In this case, as the PV production is higher than the committed power, the ESS starts to charge with both strategies. The basic one charges the ESS till the moment it is completely full, and from that moment onwards power is directly supplied from the PV panels to the grid. On the other hand, the MPC system detects in advance that there exists a surplus of power production and, as the imbalance prices decrease along that intraday session, the system releases part of that surplus before the price changes at $12 \mathrm{~h}$ and once again at $13 \mathrm{~h}$. Note that the 

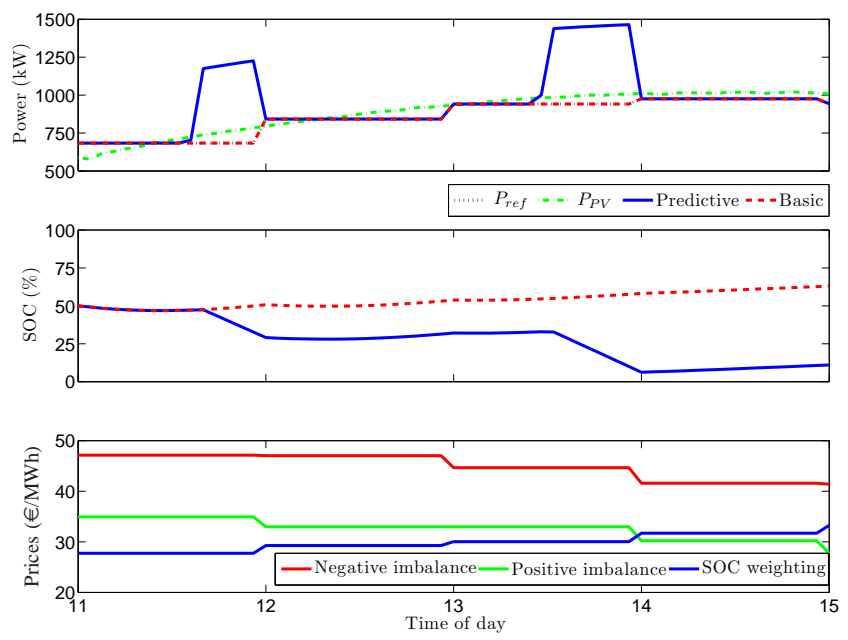

Fig. 7. Accurate estimation of PV production case study.

MPC does not start selling this excess of power before (e.g. at $11 \mathrm{~h}$ ), what would allow a smoother power profile, because at that point the prediction does not indicate yet that some energy is going to be left over. Contrary to the previous case, as the energy during the control horizon is valued at a positive imbalance price, there arrives a time (at $14 \mathrm{~h}$ ) at which storing energy in the ESS is valued higher than if it was sold, i.e. $c_{\text {soc }}(t+N+1)>c_{p}(t)$. From this point, the optimal strategy involves charging the ESS.

The total economic revenue (16) is in this case $2.35 \%$ higher if the MPC is implemented.

This simulation, which covered a 4-hour long intraday market session, lasted a total of $217 \mathrm{~s}$ with an average and a maximum computation time for the optimizations of, respectively, $3.32 \mathrm{~s}$ and $3.81 \mathrm{~s}$.

\section{Accurate estimation of $P V$ production}

Lastly, as a third case study, we analyze the situation for a sunny summer day in which the PVGIS model and the actual PV production are very close and the power commitments are well defined. The working period is again the fifth intraday market session and the ESS is initially at 50\% SOC. The simulation, which is performed in $206.7 \mathrm{~s}$ (3.33 s average and $3.59 \mathrm{~s}$ maximum computation times for the optimizations), offers the results shown in Fig. 7.

It can be seen how the MPC feeds an excess of energy to the grid just before $12 \mathrm{~h}$, when it is best paid, and once again just before $14 \mathrm{~h}$, at the cost of leaving the ESS almost empty. This is due to the low $c_{s o c}$ compared with $c_{p}(t)$ in those moments. After $14 \mathrm{~h}$ this situation reverts and the ESS starts to charge. According to (16), the MPC provides a revenue $1.57 \%$ higher. However, it is important to note that although this is the optimal strategy according to the optimization index, in this case one might prefer a most conservative approach. This way, as the energy surplus is paid at a positive imbalance price, it might be considered more effective to save a higher amount of energy to give some more flexibility to the system in order to cope with an unexpected overestimation of future PV production. Such behavior can be accomplished by tuning the MPC with a higher $c_{s o c}$ in order to give more relevance to the stored energy.

\section{CONCLUSION}

PV systems can mitigate their production variability when they employ ESS. As these technologies are still considerably expensive, installations need to be designed with an accurate ESS rating. However, when a reduced capacity is implemented, the ESS is more likely to saturate, which turns into failure of the power commitment tracking and economic penalties. In this sense, the implementation of an MPC controller in a PV+ES system has clear benefits, as it allows reducing penalties associated to imbalances. This is mainly due to the fact that the predictive control strategy has at its disposal a more accurate prediction of the PV production than the system had when the day ahead bid in the daily market. This helps to detect in advance when the ES is going to saturate and, if the imbalance costs are known, shift the tracking failure to the moment in time when it produces the minimal economic penalties. Therefore, the strategy here proposed improves the integration of intermittent energy sources with ES that participate in an electricity market. Although generators in countries such as Spain receive feed-in tariffs instead of participating in such a market, they are anyway charged for their imbalances, so it would also be helpful for them.

The MPC technique has been tested in simulation with actual irradiation data from a PV installation located in Seville (Spain). Furthermore, the computational cost of the solved optimization problems (LP) have been shown to be low enough to allow the implementation of the MPC strategy in a real plant with a 4-minute sampling time.

Lastly, the use of an advanced optimization similar to the one implemented in the MPC would allow a PV generator to submit bids into the day ahead market that try to maximize profits (i.e. higher generation in peak hours) while it avoids ESS saturation.

\section{REFERENCES}

[1] M. I. Hoffert, K. Caldeira, G. Benford, D. R. Criswell, C. Green, H. Herzog, A. K. Jain, H. S. Kheshgi, K. S. Lackner, and J. S. Lewis, "Advanced technology paths to global climate stability: energy for a greenhouse planet," Science, vol. 298, pp. 981-987, Nov. 2002.

[2] B. Bose, "Global warming: Energy, environmental pollution, and the impact of power electronics," IEEE Ind. Electron. Mag., vol. 4, pp. 617, Mar. 2010.

[3] REN21, Renewables 2011 Global Status Report. Paris: REN21 Secretariat, 2011.

[4] D. A. Halamay, T. K. A. Brekken, A. Simmons, and S. McArthur, "Reserve requirement impacts of large-scale integration of wind, solar, and ocean wave power generation," IEEE Transactions on Sustainable Energy, vol. 2, pp. 321-328, Jul. 2011.

[5] T. Muneer, S. Younes, and S. Munawwar, "Discourses on solar radiation modeling," Renewable and Sustainable Energy Reviews, vol. 11, pp. 551-602, May 2007.

[6] C. A. Gueymard, "The sun's total and spectral irradiance for solar energy applications and solar radiation models," Solar Energy, vol. 76, pp. 423453, Apr. 2004.

[7] J. Marcos, L. Marroyo, E. Lorenzo, and M. García, "Smoothing of PV power fluctuations by geographical dispersion," Progress in Photovoltaics: Research and Applications, vol. 20, pp. 226-237, Mar. 2012.

[8] P. Denholm and R. M. Margolis, "Evaluating the limits of solar photovoltaics (PV) in electric power systems utilizing energy storage and other enabling technologies," Energy Policy, vol. 35, pp. 4424-4433, Sep. 2007. 
[9] Y. Riffonneau, S. Bacha, F. Barruel, and S. Ploix, "Optimal power flow management for grid connected pv systems with batteries," IEEE Transactions on Sustainable Energy, vol. 2, pp. 309-320, Jul. 2011.

[10] R. Sioshansi and P. Denholm, "The value of concentrating solar power and thermal energy storage," IEEE Transactions on Sustainable Energy, vol. 1, pp. 173-183, Oct. 2010.

[11] R. Hara, H. Kita, T. Tanabe, H. Sugihara, A. Kuwayama, and S. Miwa, "Testing the technologies," IEEE Power Energy Mag., vol. 7, pp. 77-85, May-Jun. 2009.

[12] S. Teleke, M. E. Baran, S. Bhattacharya, and A. Q. Huang, "Rule-based control of battery energy storage for dispatching intermittent renewable sources," IEEE Transactions on Sustainable Energy, vol. 1, pp. 117-124, Oct. 2010.

[13] J. P. Barton and D. G. Infield, "Energy storage and its use with intermittent renewable energy," IEEE Trans. Energy Convers., vol. 19, pp. 441-448, Jun. 2004

[14] S. Vazquez, S. M. Lukic, E. Galvan, L. G. Franquelo, and J. M. Carrasco, "Energy storage systems for transport and grid applications," IEEE Trans. Ind. Electron., vol. 57, pp. 3881-3895, Dec. 2010.

[15] H. Beltran, M. Swierczynski, A. Luna, G. Vazquez, and E. Belenguer, "Photovoltaic plants generation improvement using Li-ion batteries as energy buffer," in Proc. IEEE Int. Symposium on Industrial Electronics, Gdansk, Poland, 2011, pp. 2063-2069.

[16] H. Fakham, D. Lu, and B. Francois, "Power control design of a battery charger in a hybrid active PV generator for load-following applications," IEEE Trans. Ind. Electron., vol. 58, pp. 85-94, Jan. 2011.

[17] H. Kanchev, D. Lu, F. Colas, V. Lazarov, and B. Francois, "Energy management and operational planning of a microgrid with a PV-based active generator for smart grid applications," IEEE Trans. Ind. Electron., vol. 58, pp. 4583-4592, Oct. 2011.

[18] N. Kakimoto, H. Satoh, S. Takayama, and K. Nakamura, "Ramp-rate control of photovoltaic generator with electric double-layer capacitor," IEEE Trans. Energy Convers., vol. 24, pp. 465-473, Jun. 2009.

[19] M. Uzunoglu, O. C. Onar, and M. S. Alam, "Modeling, control and simulation of a PV/FC/UC based hybrid power generation system for stand-alone applications," Renewable Energy, vol. 34, pp. 509-520, Mar. 2009.

[20] M. Lafoz, L. Garcia-Tabares, and M. Blanco, "Energy management in solar photovoltaic plants based on ess," in 13th EPE-Power Electronics and Motion Control Conf., 2008, pp. 2481-2486.

[21] L. M. Costa, F. Bourry, J. Juban, and G. Kariniotakis, "Management of energy storage coordinated with wind power under electricity market conditions," in Proc. 10th Int. Conf. Probabilistic Methods Applied to Power Systems, Rincón, Puerto Rico, May 2008, pp. 1 -8.

[22] M. Dicorato, G. Forte, M. Pisani, and M. Trovato, "Planning and operating combined Wind-Storage system in electricity market," IEEE Transactions on Sustainable Energy, vol. 3, pp. 209 -217, Apr. 2012.

[23] L. Xie and M. D. Ilić, "Model predictive dispatch in electric energy systems with intermittent resources," in IEEE Int. Conf Systems, Man and Cybernetics, 2008, pp. 42-47.

[24] L. Xie and M. Ilić, "Model predictive economic/environmental dispatch of power systems with intermittent resources," in IEEE Power and Energy Society General Meeting, 2009, pp. 1-6.

[25] M. Khalid and A. Savkin, "A model predictive control approach to the problem of wind power smoothing with controlled battery storage," Renewable Energy, vol. 35, pp. 1520-1526, Jul. 2010.

[26] E. F. Camacho and C. Bordons, Model Predictive Control. Springer, 2004.

[27] J. A. Rossiter, Model-Based Predictive Control: A Practical Approach. CRC Press, 2003.

[28] F. Borrelli, Constrained optimal control of linear and hybrid systems. Springer Verlag, 2003.

[29] European Commission. Joint Research Centre, "PVGIS database." [Online]. Available: http://re.jrc.ec.europa.eu/pvgis/index.htm

[30] H. Beltran, E. Pérez, N. Aparicio, and P. Rodriguez, "Daily solar energy estimation for minimizing energy storage requirements in PV power plants," unpublished.

[31] R. Perez, P. Ineichen, K. Moore, M. Kmiecik, C. Chain, R. George, and F. Vignola, "A new operational model for satellite-derived irradiances: description and validation," Solar Energy, vol. 73, pp. 307-317, Nov. 2002.

[32] M. Thekaekara, R. Kruger, and C. Duncan, "Solar irradiance measurements from a research aircraft," Appl. Opt., vol. 8, pp. 1713-1732, Aug. 1969.

[33] R. J. Doviak and D. S. Zrnic, Doppler radar and weather observations, 2nd ed. Academic Press, 1993.
[34] Red Eléctrica de España, "Sistema de Información del Operador del Sistema Eléctrico Español." [Online]. Available: http://www.esios.ree.es

[35] K. Fukuda and A. Prodon, Double description method revisited. Springer, 1996.

[36] K. Fukuda, $c d d / c d d+$ Reference Manual, Institute for Operations Research, ETH-Zentrum, 1997.

[37] M. Kvasnica, P. Grieder, M. Baotić, and M. Morari, Multi-parametric toolbox (MPT). Springer, 2004. 